Rev. Chit. Pediatr. 59 (2); 105-107, 1988

\title{
Evaluación de un método de rastreo auditivo en escolares
}

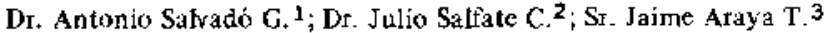

\begin{abstract}
Audioscope hearing screening
We cvaluated, the Welch Allyn audioscope to screen hearing losses in 79 children between 7 and 8 year 3 olf from an urban school. The instrument allows both audiometric testing (at $500,1000,2000,4000 \mathrm{~Hz}$, at $25 \mathrm{~dB}$ hearing leve1) and otoscopic examination. Children were assesed inmediately after evaluation with a traditional audiometer from our hospital. In $9 / 79$ children we found a variety of hearing losses, eight of which were identifjed by the audioscope. The audioscope was as accurate as the audiometer in identifying children with normal hearing and those with hearing loss, although it may produce a high proportion of false positive results. To reduce this highf false positive rate, it is recomended repeated screening to be conducted inmediately at ter the fir st. The aparatus is easy to use and more precise than other previous screening methods.

(Key words: hearing loss. scicening, audioscopc).
\end{abstract}

Durante el control de salud escolar se evalúa entre otros parámetros la capacidad auditiva, ya que por lo menos 3 a $4 \%$ de la población escolar sufre déficit de aưdición. ${ }^{1,2}$

Tradicionalmente se ha utilizado en nuestro medio la prueba de la "voz cuchicheada", ${ }^{\text {prue- }}$ ba poco precisa y subjetiva, con lo cual las

1. Médico pediatra Hospital Regional de Antofagasta.

2. Médico otorrinolaringólogo, Hospital Regional de Antofagasta.

3. Fonoaudiólogo, Hospital Regional de Antofagasta. referencias a los servicios de otorrinolaringología se ven sobrecargadas, pudiendo además pasar por alto a niños con déficit auditivo importante. En este último caso, las consecuencias pueden ser serias para el desarrollo del lenguaje. creando desajuste social e impedimento intelectual.

La causa más común de déficit auditivo la constituyen los trastornos de tipo conductivo asociados a enfermedad de oído medio. Se debe por lo tanto, identificar precozmente estos trastornos, ojalá antes de los cuatro años de edad.

La prueba de elección para rastreo es la audiometría de tonos puros, estimándose que 
con este métado $5 \%$ o más de los nifios presentan resultados anor males. 3

En el presente trabajo evaluamos la aplicación de un nuevo método de rastreo para defectos de la audición, en escolares, evaluado con exxito recientemente 4.5 que involucra una audiometría de tonos puros.

\section{MATERIAL Y METODO}

Se practicó evaluación auditiva a 79 niños de ambos sexos, de 7 a 8 años de edad, de una escuela básica del area urbana de Antofagasta, en el período de marzo a agosto de 1986, mediante un audioscopio (Audios cope Wolch Allyn Inc., Skaneateles Falls, N.Y., U.S.A.) (figura $){ }^{6}$ instrumento que incorpora, a un otoscopio dc fibra óptica tradicional, un dispositivo que emite secuencialmente sonidos de tonos puros a $500 \mathrm{~Hz}, 1000 \mathrm{~Hz}$, $2000 \mathrm{~Hz}$ y $4000 \mathrm{~Hz}$, a una intensidad de $25 \mathrm{~dB}$ permitiendo además visualizar el conducto auditivo ex terno $y$ el tímpano.

Se instruyó a los niños para que señalaran cada vez que escuchaban una emisión de sonido, los que eran producidos a intervalos de 1,5 scgundos y con una duración similar. Ambos oídos fuer on evaluados de igual forma. Si el nifó fallaba en señalar una frecuencia, el procedimiento se repitió dos o tres veces para confirmar

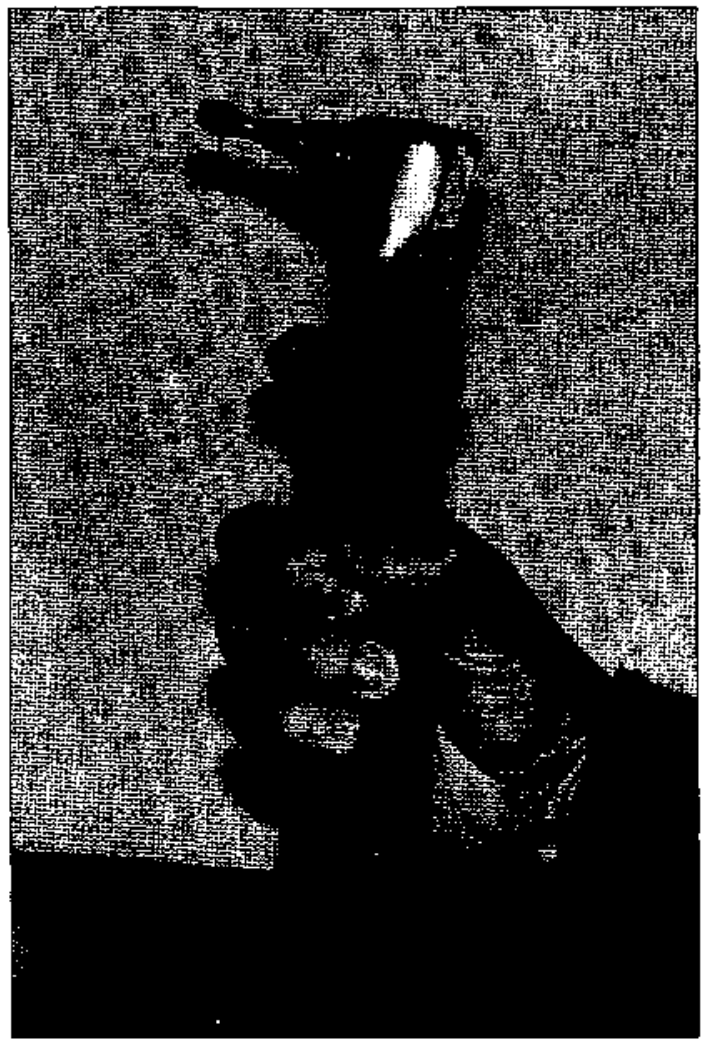

el déficit. Se consideró normal el resultado cuando el niño falló en dos o más frecuencias en forma reiterada.

Como patrón de referencias, les fue practicada minutos despues una audiometria tradicional, utilizando un audiómetro Madsen 08822 (Madsen Electronics, Buffalo, N.Y. U.S. A.), aplicando las mtsmas intensidades y frecuencias del método de rastreo. Ambos procedimientos fueron realizados en la cámara silente de nuestro hospital.

\section{RESULTADOS}

En 9/79 niños se detectó hipoacusia. Esta fue identificada en $8 / 9$ con el audioscopio, y confirmada en todos con audiometría. En un caso con evidencia audiométrica de hipoacusia, $\mathbf{a}$ evaluación por audioscopio dió un resultado falso negativo. Sin embargo, este caso correspondía a una otitis supurada e igualmente fue diagnosticada y tratada. Considerando este hecho, ningún niño con trastorno auditivo fue excluido por el audioscopio.

En los nueve niños había una afección auditiva: otitis serosa $(n=4)$, otitis supurada $(n=2)$, tapón de cerumen $(n=2), y$ disfusión tubaria

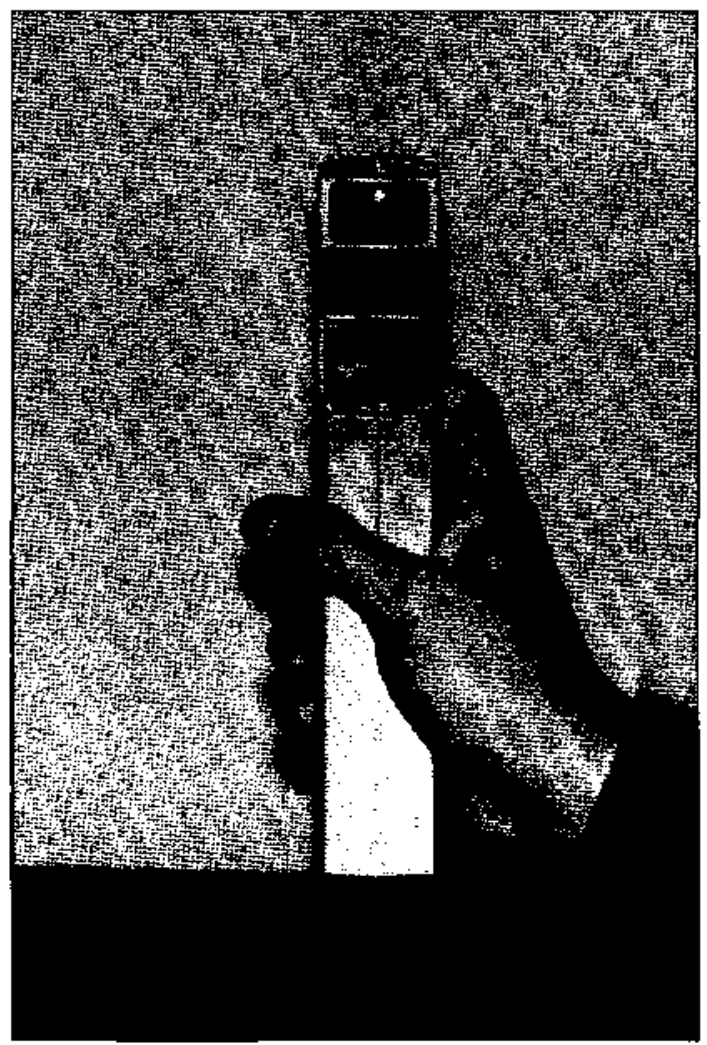

Figura Aspecto lateral y dorsal del audioscopio (AudioScope, Welch Allyn Inc.) 
$(n=1)$. En once casos el audioscopio dió resultados positivos para sonidos de frecuencias bajas, sin embargo la audiometría fue normal (falsos positivos).

La sensibilidad global del método fue de $89 \%$ y la especificidad alcanzó a 84\%. El valor predictivo positivo, es decir la frecuencia con que el déficit auditivo estaba presente cuando el resultado era normal, fue de $42 \%$, siendo la eficiencia global del método (frecuencia con que el audioscopio predijo correctamente) de $84,8 \%$.

\section{DISCUSION}

E] procedimiento recomendado actualmente para rastreo auditivo es la audiometría de tonos puros, con sonidos de frecuencias de 1000,2000 y $4000 \mathrm{~Hz}$, aplicando tonos de $500 \mathrm{~Hz}$ sólo cuando el ambiente lo permita; el ruido ambiental de be ser siempre $\leqslant 46 \mathrm{~dB} .^{7}$

Nuestros resultados muestran que el audios. copio puede ser utilizado eficientemente en escolares chilenos. La sensibilidad debe ser conside. rada excelente, especialmente si aceptamos que se trata de un método de evaluación inicial.

Recientemente se han comunicado resultados sunilares a los nuestros en cuanto a sensibilidad y especificidad en un grupo de niños de seis años. ${ }^{5}$

Un grupo importante de niños presentó resuj. tados falsos positivos, pero sólo para frecuencias bajas, especialmente $500 \mathrm{~Hz}$, lo que se explica por el ruido ambiental residual dentro de la cámara silente, que alcanzaba entre 30 y $40 \mathrm{~dB}$. Otra razón para explicar los resultados falsos positivos, es que el audioscopio, a diferencia de audiómetro no permite "presentar" un nivel de intensidad mayor para la prueba de las frecuencias iniciales (500 y $1000 \mathrm{~Hz}$ ), lo que dificulta el aprendizaje del método por parte del niño. Con cl fin de reducir la tasa de falsos positivos, se recomienda repetir el procedimiento una o más veces. E1 audioscopio cs un medio fácil de utilizar, consume poco tiempo, su costo es aceptable y puede ser utilizado en habitaciones con algún grado de ruido residual.

\section{RESUMEN}

En 79 nifios de 7 y 8 años de edad se rastreó hipoacusia mediante un audioscopio. El aparato consiste en un otoscopio tradicional de fibra óptica unido a un dispositivo para emitir sonidos de tono puro de $25 \mathrm{~dB}$ en un rango de frecuencias de 500 a $4.000 \mathrm{~Hz}$. Los niños fueron estudiados inmediatamente después de una audiometria convencional. En 9 nifnos se encontraron dêficits de audición, 8 fueron correctamente identificados con el audioscopio. La proporción de falsos positivos puede ser muy alta, para reducirla se recomienda reiterar el procedimiento cuando el primer resultado sugiere un defecto. El equipo es de costo razonable, fácil de usar y más preciso que los métodos simples de rastreo semiológico.

\section{AGRADECIMIENTOS}

A la señora Gladys Sinchez, Directora de la Jiscuela D-74 de Antofagasta, por su colaboración en la realización de este trabajo.

\section{REFERENCIAS}

1. Patri M.A.: Salud Escolar. lixumen de agudeza audi. tiva. Santiago. Ld. Andrés Bello 1979; 114.

2. National Center for Heath Statistics. Survey. U.S.A 1977.

3. Anterican Acodemy of Pediatrics. School Health: A Guide for Health Professionals, 1981. Appendix C. Hearing Screcning.

4. Gershell J., Kruger B., Giraudi-Perry D.: Accuracy of the WeIch Allyn Audioscope and Traditional Hearing Sarening for children with known hearing loss. J Pedjatr $1985 ; 106: 15-20$.

S. Orbando M.S., Frank T.i Audiometer and AudioScope hearing screening compared with threshold test in young children. I Pediatr 1987; 110:261-263.

6. AisdioScope Welch Allyn. Manual de Operación.

7. American Speechand Hearing Association: Guide. lines for identification audionctry. ASHA, 1975 . 17: 94 . 